\title{
The Genotype MTBDRplus ver. 2.0 test as a quick indicator of resistance to rifampicin and isoniazid in Mycobacterium tuberculosis strains
}

Salvatore Nisticò', Paola Magno', Annelisa Borelli',Vincenzina Caruso', Vilma Villella', Lorenzo Antonio Surace ${ }^{2}$, Laura Pontoriero², Domenico Lucchino³, Giuseppina Berardelli', Ginafranco Griffo ${ }^{3}$, Domenico Caruso', Rosa Anna Leone'

I Laboratorio di Microbiologia e Virologia, P.O. di Lamezia Terme A.S.P. di Catanzaro

2 Dispensario Funzionale Aziendale A.S.P. di Catanzaro

3 U.O. di Malattie Infettive, P.O. di Lamezia Terme A.S.P. di Catanzaro

Key words: Tuberculosis, Antitubercular drugs, Multidrug resistant, Genotype MTBDRplus

\begin{abstract}
Genotype MTBDR plus ver 2.0: indicatore rapido di resistenza a Rifampicina ed Isoniazide in ceppi di Mycobacterium tuberculosis
\end{abstract}

\section{SUMMARY}

Tuberculosis is still a global emergency and a major public health problem, in some cases related to the appearance of strains of multi drug resistance (MDR) and extensive drug resistance (XDR) Mycobacterium tuberculosis complex. The correct determination of antibiotic sensitivity profiles is therefore crucial to carry out appropriate treatment aimed to decrease the infectivity of each patient and to reduce mortality. The poor adherence to treatment by the patient or the use of therapies based on a single drug, as a result of incorrect requirements, promote the development of drug-resistance. Have some time on the market of molecular diagnostic tests that allow, quickly and directly from biological sample to search for resistance genes some key drugs of anti-TB therapy (Rifampicin and Isoniazid). One of the tests in question is the Genotype MTBDRplus ver 2.0 which can reveal the presence of genes for resistance to Isoniazid (INH) and Rifampin (RMP). The loci analyzed are those corresponding to the $r p o B$ gene for rifampicin, kat $G$ and inhA for isoniazid. Our study is based on the analysis of 83 strains of tubercular Mycobacteria identified and isolated from patients with tuberculosis disease and subjected to the tests sensitivity, searching for mutations and phenotypic susceptibility testing for Rifampicin and Isoniazid. The comparison of the results has shown that the results obtained using the Genotype MTBDRplus ver 2.0 test, were similar to the results obtained by the traditional susceptibility testing.

\section{INTRODUZIONE}

La malattia tubercolare rappresenta una emergenza mondiale ed un importante problema di salute pubblica $(4,8)$. Il bacillo tubercolare ha una resistenza naturale a molti dei farmaci antibiotici in uso ed il numero delle molecole disponibili per la terapia è esiguo $(2,5,7)$. Sempre più frequentemente sono isolati ceppi batterici multi-farmaco resistenti (MDR), resistenti ad isoniazide e rifampicina, e ceppi con farmaco resistenze estese anche agli antibiotici di seconda linea terapeutica (XDR), resistenti oltre che ad isoniazide e rifampicina, anche a fluorochinoloni e ad almeno uno dei farmaci iniettivi tra amikacina, kanamicina, capreomicina, viomicina $(3,6)$. Questo problema induce a raccomandare l'esecuzione dell'antibiogramma non solo su tutti i ceppi di nuovo isolamento, ma anche sui ceppi isolati dall'esame colturale di pazienti in terapia farmacologica da oltre

\section{2-3 mesi $(8,9)$.}

Nei micobatteri tubercolari le resistenze farmacologiche sono da attribuire a mutazioni cromosomiche; è, tuttavia, possibile lo sviluppo di resistenze correlate all'inserzione di elementi genici mobili, che inattivano geni critici per l'azione del farmaco (1). Sono disponibili in commercio test che ricercano mutazioni note presenti sul gene $r p o B$ per la resistenza a rifampicina e sui geni $k a t G$ e $i n h A$ per la resistenza ad isoniazide.

La rapida identificazione di queste mutazioni consente di attuare un appropriato regime terapeutico, limitando la diffusione di ceppi MDR ed XDR. Lo scopo del presente lavoro è verificare se esiste concordanza tra $\mathrm{i}$ dati ottenuti utilizzando il test Genotype MTBDRplus ver 2.0, che ricerca la presenza di resistenze genetiche note a due farmaci cardine della terapia antitubercolare, rifampicina ed isoniazide e $\mathrm{i}$ dati forniti dall'antibiogramma

\section{Corresponding author: Salvatore Nisticò}

Laboratorio di Microbiologia e Virologia, P.O. di Lamezia Terme (CZ)

Tel.: 096820876I; Fax: 0968463 I I3

E-mail: snistico I@virgilio.it 
tradizionale, eseguito sugli stessi ceppi batterici. Inoltre, abbiamo voluto calcolare il vantaggio, in termini di tempi diagnostici, ottenuto ricercando le mutazioni con il test molecolare rispetto all'antibiogramma.

\section{MATERIALI E METODI}

Nel biennio 2011-2012 sono stati analizzati nel settore di Micobatteriologia dell'Unità Operativa di Microbiologia e Virologia - Presidio Ospedaliero di Lamezia Terme (CZ), 2820 campioni biologici provenienti da pazienti con sospetto di malattia tubercolare. Tali campioni sono stati sottoposti a:

Decontaminazione e concentrazione, eseguita con il sistema MycoPrep (Becton-Dickinson);

Esame microscopico per la ricerca di bacilli acido-alcool resistenti (BAAR), allestito in doppio dal sedimento del campione e colorato con Auramina (TB auramine stain Becton-Dickinson); Esame colturale, eseguito seminando parte del sedimento su due diversi terreni, uno solido (Löwenstein-Jensen, Becton-Dickinson) ed uno liquido (MGIT, Becton-Dickinson). I tubi di Löwenstein-Jensen sono stati incubati a $37^{\circ} \mathrm{C}$ per un tempo massimo, in assenza di crescita batterica, di 8 settimane. Il terreno MGIT è stato incubato nello strumento semi-automatico Bactec MGIT 960 per un tempo massimo, in assenza di crescita, di 54 giorni;

Ricerca diretta di genoma di MTC da campione biologico, eseguita utilizzando il sistema ProbeTec ET (Becton-Dickinson).

Sui campioni risultati positivi all'esame batterioscopico per BAAR o alla ricerca diretta da campione biologico di DNA sono stati eseguiti, direttamente dal sedimento, i seguenti test:

Identificazione di specie dei micobatteri appartenenti al MTC, utilizzando il test Genotype MTBC (Hain- distribuito in Italia da Arnika), un test di diagnostica molecolare, basato sulla tecnologia dell'ibridazione inversa di amplificati;

Ricerca di mutazioni genetiche note ai farmaci Rifampicina ed Isoniazide, utilizzando il sistema GenoType MTBDRplus ver 2.0 (Hain, distribuito in Italia da Arnika), test basato sulla tecnologia dell'ibridazione inversa di amplificati.

Antibiogramma per i farmaci di prima linea terapeutica, allestito dai ceppi ottenuti tramite esame colturale, utilizzando il sistema Bactec MGIT 960 SIRE kit (Becton-Dickinson).

Valutazione Esterna di Qualità (VEQ). Programma Esterno di Valutazione di Qualità INSTAND (Istituto INSTAND ev - Dusseldorf Germania). Spedizioni effettuate due volte all'anno, nel mese di febbraio e di settembre.

Ogni spedizione è composta da: cinque campioni biologici per l'esame colturale; cinque campioni biologici per la ricerca di genoma di MTC;

sei vetrini per la ricerca di BAAR; cinque ceppi per l'identificazione di specie; cinque ceppi di MTC per l'esecuzione dell'antibiogramma per: isoniazide, rifampicina, etambutolo, streptomicina, pirazinamide.

\section{RISULTATI}

Negli anni 2011 e 2012 il laboratorio di Micobatteriologia dell'Unità Operativa di Microbiologia e Virologia del Presidio Ospedaliero di Lamezia Terme ha partecipato a Valutazione Esterna di Qualità ed ha ottenuto tutte le certificazioni previste. Nello stesso periodo di tempo sono stati isolati 83 ceppi di micobatteri tubercolari da diversi materiali biologici di pazienti affetti da malattia tubercolare.

Il 37\% dei pazienti erano di nazionalità italiana, il $40 \%$ provenivano dall'Europa Sud-Centro Orientale, il 18\% dall'Africa sub-sahariana, il 4\% dal Sud-Est asiatico e dall'Asia Meridionale e $1 ' 1 \%$ dalla Russia.

In riferimento alla sede dell'infezione tubercolare, 80 pazienti erano affetti da TBC polmonare, 2 da $\mathrm{TBC}$ renale e 1 da TBC pleurica. L'età dei pazienti era compresa tra 3 e 91 anni. In riferimento al sesso, il $69 \%$ dei pazienti era di sesso maschile ed il $31 \%$ di sesso femminile. Nessuno degli 83 pazienti risultava affetto da HIV. In riferimento ai test eseguiti sugli 83 campioni, si sono ottenuti i seguenti risultati:

esame microscopico: 64 sedimenti positivi per BAAR e 19 sedimenti negativi;

test di amplificazione genica: presenza di DNA di MTC in 76 sedimenti dei campioni in esame ed assenza di materiale genetico specifico in 7 sedimenti;

esame colturale positivo in tutti gli 83 campioni; identificazione di specie: 80 ceppi di M. tuberculosis, 2 ceppi di $M$. africanum, isolati da un paziente di sesso maschile proveniente dal Senegal e da un paziente di sesso maschile proveniente dal Burkina Faso ed 1 ceppo M. bovis subsp bovis, isolato da un paziente di sesso maschile proveniente dal Togo;

ricerca genetica di mutazioni associate a resistenza farmacologica: assenza di mutazioni in 73 ceppi, 8 ceppi con resistenza ad isoniazide e 2 ceppi con resistenza a rifampicina. La resistenza a rifampicina dei 2 ceppi di $M$. tuberculosis in esame era riconducibile ad una mutazione nel codone $531(\mathrm{~S} 531 \mathrm{~L})$ del gene rpoB; i pazienti da cui sono stati isolati i due ceppi erano due donne provenienti dalla Romania. La resistenza all'isoniazide degli 8 ceppi di $M$. tuberculosis, era corre- 
lata a una mutazioni nel codone 315 (S315T1) del gene katG. In riferimento alla nazionalità dei pazienti infettati da questi 5 ceppi, due pazienti, uno di sesso maschile e uno di sesso femminile provenivano dalla Romania, uno di sesso maschile proveniva dalla Repubblica delle Filippine, uno di sesso maschile proveniva dalla Russia e uno di sesso femminile di nazionalità italiana. La mutazione nel codone $15(\mathrm{C} 15 \mathrm{~T})$ del gene inhA è stata rivelata in 3 ceppi provenienti da un paziente di sesso maschile e uno di sesso femminile di nazionalità italiana e uno di sesso maschile proveniente dalla Romania. Nessun dei ceppi è risultato MDR.

Antibiogramma: sensibilità e resistenza, dei ceppi in esame, ai farmaci isoniazide e rifampicina sovrapponibili a quelli ottenuti con il test Genotype MTBDRplus, in dettaglio: 73ceppi sensibili sia a rifampicina che ad isoniazide, 8 ceppi resistenti ad isoniazide e 2 ceppi resistenti a rifampicina.

\section{DISCUSSIONI E CONCLUSIONI}

Dall'analisi e dal confronto dei dati si evince una totale concordanza dei risultati ottenuti con l'antibiogramma e con la ricerca di mutazioni genetiche associate a resistenza ai farmaci isoniazide e rifampicina. Il test Genotype MTBDRplus ver 2.0 è eseguibile direttamente, ed in poche ore, da sedimento del campione biologico positivo per BAAR o da sedimento positivo per genoma di MTC. Pertanto, è capace, in tempi abbastanza brevi, di fornire preziose informazioni circa il possibile comportamento del ceppo in esame a due importanti farmaci antitubercolari. Per poter dimostrare i vantaggi sono stati calcolati i tempi trascorsi tra il giorno di esecuzione del test Genotype MTBDRplus ver 2.0 ed il giorno in cui si sono ottenuti i risultati dell'antibiogramma condotto con il sistema semiautomatico Bactec MGIT 960. Il guadagno di tempo risulta importante e si sono potute effettuare correzioni terapeutiche nel giro di poco tempo dall'inizio della terapia standard. Nello specifico, dai dati in nostro possesso, è stato possibile, eseguendo il Genotype MTBDRplus ver 2.0 rivelare resistenze a rifampicina o isoniazide mediamente 15 giorni prima rispetto alle stesse resistenze individuate dall'antibiogramma. È stato possibile, inoltre, differenziare tale vantaggio considerando tre diverse condizioni diagnostiche:

Esame microscopico positivo, Test di amplificazione genica positivo, Esame colturale positivo; Esame microscopico negativo, Test di amplificazione genica positivo, Esame colturale positivo; Esame microscopico negativo, Test di amplificazione genica negativo, Esame colturale positivo. Per ognuna di queste condizioni sono stati calcolati il tempo trascorso dall'arrivo del campione in laboratorio all'esecuzione del test Genotype MTBDR plus ver 2.0 ed il tempo trascorso dall'arrivo del campione in laboratorio ed i risultati ottenuti dall'antibiogramma condotto con il sistema semiautomatico Bactec MGIT 960.

I tempi sono stati calcolati separatamente per l'anno 2011 e per l'anno 2012. Il motivo di questa scelta è dovuto alle modifiche effettuate nella metodica del test Genotype MTBDR plus ver 2.0, nel 2011 prevedeva l'esecuzione dell'esame solo da microscopia positiva per BAAR, mentre dal 2012 la metodica ha permesso l'esecuzione del test anche da campione con esame microscopico negativo, ma con ricerca diretta di genoma di MTC positiva. Nel 2011 il tempo guadagnato è stato mediamente di 18 giorni, nel 2012, grazie alla possibilità di eseguire il test genetico anche da BAAR negativo ed amplificazione genica positiva, il tempo guadagnato è stato mediamente di 21 giorni.

Le tre condizioni prese precedentemente in esame ed i tempi medi sono schematizzati nella Tabella 1.

Esaminando la Tabella 1 è possibile fare diverse valutazioni.

Per una tempestiva ed agevole diagnosi di TBC, la condizione maggiormente favorevole è quella in cui il campione presenta $\mathrm{EM}+, \mathrm{AG}+$ e $\mathrm{EC}+$, il ché indica un'elevata carica batterica del campione. Nell'anno 2011 i tempi medi sono stati di 5 giorni per il Genotype MTBDRplus ver 2.0 e di 20 giorni per l'antibiogramma tradizionale, mentre nel 2012 sono stati di 1 giorno per la ricerca delle resistenze genetiche e di 18 giorni per l'antibiogramma.

Tabella I. Tempi necessari per ottenere informazioni terapeutiche con Genotype MTBDR plus e con l'antibiogramma tradizionale.

\begin{tabular}{lcccc}
\hline & \multicolumn{2}{c}{ ANNO 20 I I } & \multicolumn{2}{c}{ ANNO 20 I 2 } \\
\hline EM+/AG+/EC+ & Genotype & Bactec & Genotype & Bactec \\
\hline EM-/AG+/EC+ & 5 giorni & 20 giorni & I giorno & I8 giorni \\
\hline EM-/AG-/EC+ & 12 giorni & 3 I giorni & 3 giorni & 36 giorni \\
\hline
\end{tabular}

$\overline{\mathbf{E M}}+/-$ = Esame microscopico positivo /negativo AG +/- = Amplificazione genica positiva $/$ negativa $\mathbf{E C}+/-=$ Esame colturale positivo/negativo. 
I campioni con EM-, AG+ e EC+ rappresenta la condizione moderatamente favorevole, in quanto la carica micobatterica del campione è tale da poter essere rilevata dal test di AG, ma insufficiente per l'EM. I risultati del Genotype MTBDRplus ver. 2.0 sono stati ottenuti nel 2011 dopo 12 giorni dall'arrivo del campione mentre nel 2012 dopo 3 giorni; i risultati dell'antibiogramma fenotipico sono pervenuti nel 2011 dopo 31 giorni dall'arrivo del campione e nel 2012 dopo 36 giorni.

I campioni con EM-, AG- ed EC+ rappresentano la condizione più sfavorevole per porre diagnosi microbiologica di malattia tubercolare, in quanto la bassissima carica batterica del campione non è apprezzabile nè attraverso l'AG, tantomeno mediante EM. Per l'esecuzione dei test di sensibilità e di rivelazione di mutazioni geniche è stato, dunque, necessario attendere l'esito dell'esame colturale (su terreno solido o liquido). Sia nel 2011 che nel 2012 i risultati del Genotype MTBDRplus ver 2.0 sono stati ottenuti mediamente dopo 16 giorni dall'arrivo del campione, mentre i risultati dell'antibiogramma fenotipico sono stati ottenuti, rispettivamente, dopo 37 giorni nel 2011 e dopo 35 giorni nel 2012.

Possiamo quindi concludere che è vantaggioso eseguire i test molecolari per la ricerca di resistenze genetiche ai farmaci chiave della terapia antitubercolare e che essi possono evidenziare con precocità la presenza di eventuali ceppi con farmacoresistenze, come i temuti MDR. Non bisogna, tuttavia, dimenticare che il test Genotype MTBDR plus ver 2.0 evidenzia solo le resistenze che trovano la loro origine nelle regioni rpoB, katG e inhA, pertanto eventuali resistenze farmacologiche, che hanno per origine mutazioni situate in altri geni, non possono essere rivelate. Risulta necessario, per tutti i campioni positivi all'esame colturale, allestire sempre l'antibiogramma tradizionale. In riferimento alla possibilità di ceppi MDR, nessuno degli 83 ceppi batterici studiati ha dimostrato resistenza contemporaneamente a isoniazide e rifampicina. Tuttavia, anche la resistenza ad uno solo di questi due antibiotici comporta la modifica sostanziale dello schema terapeutico rispetto a quello standard, sia nel numero di antibiotici da usare che nella durata della terapia. L'attendibilità e la rapidità di esecuzione del test Genotype MTBDRplus ver 2.0 consente di ausiliare la dia- gnostica della tubercolosi, escludendo con elevata possibilità la presenza di eventuali ceppi resistenti, in attesa della risposta dell'antibiogramma fenotipico ed indirizzando così il clinico verso una corretta terapia. Una terapia efficace riduce notevolmente il rischio di comparsa di ceppi MDR e XDR. Anche la gestione terapeutica dei contatti di caso si avvantaggia dalle informazioni che il test Genotype MTBDRplus può precocemente fornire, nel momento in cui rivela la presenza di resistenza all'isoniazide. Tali test, per la loro peculiarità e per i loro costi, non dovrebbero essere eseguiti da tutti i laboratori che si occupano di diagnosi di tubercolosi, ma solo da Centri che hanno esperienza nella diagnostica micobatteriologica avanzata (Centri di Riferimento). In ultimo, è utile ricordare che, per poter considerare i risultati analitici validi ed attendibili, è consigliato a tutti i laboratori di micobatteriologia la partecipazione a programmi di Valutazione Esterna di Qualità.

\section{BIBLIOGRAFIA}

1. Almeda da Silva PE, Palomino JC. Molecular basis and mechanisms of drug resistance in Mycobacterium tuberculosis: classical and new drugs. J Antimicrob Chemother, 2011; 66: 1417-30.

2. A.T.S., C.D.C., I.D.S.A. Treatment of tuberculosis. 2003. MMWR 52: 1-77.

3. C.D.C. Emergence of Mycobacterium tuberculosis resistance to second-line drugs worldwide, 2000-2004. MMWR Morb Mortal Wkly Rep 2006; 55: 301-5.

4. Gandhi NR, Nunn P, Dheda K, et al. Multidrugresistant and extensively drug-resistant tuberculosis: a theat to global control of tuberculosis. Lancet 2010; 375: $1830-43$.

5. Gillespie SH. Evolution of drug resistance in Mycobacterium tuberculosis: clinical and molecular perspective. Antimicrob Agents Chemother 2002; 46: 267-74.

6. Migliori GB, De Iaco G, Besozzi G, et al. First tuberculosis cases in Italy resistant to all tested drugs. 2007. Euro Surveill 12: E070517.1

7. Richeldi L, Ewer K, Losi M, et al. T Cell-based tracking of multidrug resistant tuberculosis infection following brief exposure. Am J Respir Crit Care Med 2004; 170: 288-95.

8. Tenover FC, Crawford JT, Huebner RE, et al. The resurgence of tuberculosis. Is your laboratory ready? $J$ Clin Microbiol 1993; 31: 767-70.

9. Tortoli E, Piersimoni C, Scarparo C, Cirillo MD. Capitolo 9: Test di sensibilità 161-189. In Micobatteriologia Clinica. 2008. Selecta Medica, Pavia. 